\title{
Homogeneous Balitsky-Kovchegov hierarchy and Reggeon field theory
}

\author{
N. V. Prikhod'ko* \\ Yaroslav-the-Wise Novgorod State University (NovSU), Veliky Novgorod \\ E-mail: nikolai.prikhodko@novsu.ru
}

The low dimensional field theory corresponding to Balitsky-Kovchegov hierarchy of equations with homogeneous initial conditions is derived using two approaches. The first approach is based on stochastic Balitsky-Kovchegov equation and uses Martin-Siggia-Rose auxiliary response fields. The second one is based on dimensional reduction of BFKL pomeron field theory action functional. Constructed field field theory has a structure of Reggeon Field Theory which is much simpler that BFKL pomeron field theory.

It is shown that conventional non-stochastic Balitsky-Kovchegov equation is a classical field equations of this field theory with specific boundary conditions. Since BFKL pomeron field theory contains both target and projectile currents it is argued that in case of scattering different class of boundary conditions should be used.

Using the boundary conditions that directly respect target-projectile duality different class of evolution equations is constructed. Analogous to non-stochastic Balitsky-Kovchegov equation it also contains additional terms which represents pomeron merging. These evolution equations allows consistent definition of various experiment related quantities such as inclusive and exclusive cross-section in case of scattering of spatially homogeneous target and projectile.

The work is supported by RFBR grant 11-02-01395-a.

XXI International Baldin Seminar on High Energy Physics Problems,

September 10-15, 2012

JINR, Dubna, Russia

${ }^{*}$ Speaker. 


\section{Introduction}

It was proposed in [1] that BFKL field theory can be obtained from stochastic version of Balitsky-Kovchegov equation [2, 3, 4] by means of action functional. However obtained field theory has rather complex structure and it is very hard to derive practical consequences from it. It is therefore interesting to check which form will have over variants of Balitsky-Kovchegov equation.

Balitsky-Kovchegov equation in coordinate space describes the dipole scattering amplitude $\mathscr{N}\left(\vec{x}_{1}, \vec{x}_{2}, Y\right)$ on some target(large nucleus). For spatially homogeneous target one could remove impact parameter dependence and work with value $\mathscr{N}\left(x_{12}, Y\right)$. It was shown in [5] that there exist evolution equation for $\mathscr{N}$ :

$$
\partial_{Y} \mathscr{N}(L, Y)=\bar{\alpha}_{s} \chi\left(-\partial_{L}\right) \mathscr{N}(L, Y)-\bar{\alpha}_{s} \mathscr{N}^{2}(L, Y)
$$

where $\bar{\alpha}_{s}=N_{c} \alpha_{s} / \pi, L=\ln \left(k^{2} / k_{0}^{2}\right)$, with $k_{0}$ some low momentum scale, e.g. $\Lambda_{Q C D}$ and $\mathscr{N}(L, Y)$ is defined through conventional dipole scattering cross sections.

$$
\mathscr{N}\left(L=\ln \left(k^{2} / k_{0}^{2}\right)\right)=\int \frac{d^{2} x_{12}}{2 \pi x_{12}^{2}} e^{-i \bar{k} \overline{x_{12}}} N\left(x_{12}, Y\right)
$$

The simplicity of non-linear term in (1.1) is a result of eikonal approximation for double dipole cross section. Without it one gets the spatial homogeneous BFKL equation.

The operator $\chi\left(-\partial_{L}\right)$ is defined by the characteristic BFKL function [6, 7] as the Mellin transform of the LO BFKL kernel in momentum space (see [8] for precise definition of $\chi$ )

$$
\chi(\gamma)=2 \psi(1)-\psi(\gamma)-\psi(1-\gamma)
$$

where $\psi(\gamma)=\frac{\Gamma^{\prime}(\gamma)}{\Gamma(\gamma)}$ is digamma function.

If one wants to get some results without eikonal approximation (1.1) one arrives to equation which contains double dipole cross section.

$$
\partial_{Y} \mathscr{N}(L, Y)=\bar{\alpha}_{s} \chi\left(-\partial_{L}\right) \mathscr{N}(L, Y)-\bar{\alpha}_{s} \mathscr{N}_{2}(L, Y)
$$

In this case equation for double dipole cross section should be written. It has form similar to (1.4) and contain triple dipole cross section and so on. This well known process gets us spatially homogeneous Balitsky-Kovchegov hierarchy.

Without nonlinear term (1.4) can be written as:

$$
\partial_{Y} \mathscr{N}(L, Y)=\bar{\alpha}_{s} \chi\left(-\partial_{L}\right) \mathscr{N}(L, Y)
$$

with corresponding solution:

$$
\mathscr{N}(L, Y)=e^{\bar{\alpha}_{s} \chi\left(-d_{L}\right) Y} \mathscr{N}(L, 0)
$$

Representing solution of (1.4) in the form

$$
\mathscr{N}(L, Y)=e^{\bar{\alpha}_{s} \chi\left(-d_{L}\right) Y} \mathscr{N}(L, 0)+\int_{0}^{Y} e^{\bar{\alpha}_{s} \chi\left(-d_{L}\right)(Y-y)} R(L, y) d y
$$


and substituting (1.7) into (1.4) following equation for $R(L, y)$ can be written

$$
R(L, y)=-\bar{\alpha}_{s} \mathscr{N}_{2}(L, y)
$$

which gives

$$
\mathscr{N}(L, Y)=e^{\bar{\alpha}_{s} \chi\left(-d_{L}\right) Y} \mathscr{N}(L, 0)-\bar{\alpha}_{s} \int_{0}^{Y} e^{\bar{\alpha}_{s} \chi(-d L)(Y-y)} \mathscr{N}_{2}(L, y) d y
$$

It is clearly seen that (1.9) resembles scattering amplitude for Reggeon field theory in space $L, Y$. Obliviously similar representation can be written for $\mathscr{N}_{2}(L, Y)$ and high dipole cross section.

It is however non oblivious how to obtain such decomposition from full Balitsky-Kovchegov hierarchy. And if we want Reggeon field theory equivalent Balitsky-Kovchegov hierarchy we should take some over way. Such method is commonly known is stochastic physics was first applied in QCD in [9].

\section{Stochastic process}

It is known that Balitsky-Kovchegov hierarchy can be obtained from some particular stochastic differential equation.

$$
\partial_{t} u(L, t)=\chi\left(-\partial_{L}\right) u(L, t)-u^{2}(L, t)+\sqrt{P(u(L, t))} \eta(L, t)
$$

where $t=\bar{\alpha}_{s} Y, u(L, t)$ is stochastic variable which averages to correlators $\mathscr{N}_{i}$ and $\eta(L, t)$ is Gaussian noise with properties:

$$
\begin{gathered}
\langle\eta(L, t)\rangle=0 \\
\left\langle\eta\left(L_{1}, t_{1}\right) \eta\left(L_{2}, t_{2}\right)\right\rangle=\delta\left(L_{1}-L_{2}\right) \delta\left(t_{1}-t_{2}\right)
\end{gathered}
$$

Particular form of functional $P(u(L, t))$ which is polynomial in $u(L, t)$ depends on how dipole merging is accounted. Three common forms mostly used:

$$
\begin{gathered}
P(u(L, t))=0 \\
P(u(L, t))=u(L, t) \\
P(u(L, t))=u(L, t)(1-u(L, t))
\end{gathered}
$$

Equation (2.4) corresponds to simple Balitsky-Kovchegov hierarchy without pomeron merging, (2.5) to Balitsky-Kovchegov hierarchy with dipole merging, (2.6) contains stochastic term which is proposed to give additional dense-dilute symmetry.

Generation functional $Z[J]$ for correlators of $u(L, t)$ in case of equation (2.1) can be written as

$$
Z[J]=\int e^{\int J(L, t) u(L, t) d t d L} \delta\left(\partial_{t} u(L, t)-\chi\left(-\partial_{L}\right) u(L, t)+u^{2}(L, t)-\sqrt{P(u(L, t))} \eta(L, t)\right) D u D \eta
$$

where we should impose Ito prescription for time discretization to remove complicated Jacobian in functional integral. Noise term can be easily integrated over and we can obtain Onsager-Machlup generating functional. However this functional is quite non linear and not easily identified with QFT. 
Previous formula can be rewritten with help of Martin-Siggia-Rose [10] auxiliary fields $u^{\dagger}(L, t)$ as (skipping unnecessary $L$ and $t$ ):

$$
Z[J]=\int e^{\int J u d t d L} e^{\left.\int u^{\dagger}\left(\partial_{t} u-\chi\left(-\partial_{L}\right) u+u^{2}-\sqrt{P(u)} \eta\right)\right) d t d L} \operatorname{DuDiu}{ }^{\dagger} D \eta
$$

Since noise $\eta$ is Gaussian integration over noise $\eta$ is rather trivial:

$$
F\left(u, u^{\dagger}\right)=\int e^{-\int u^{\dagger} \sqrt{P(u)} \eta d t d L} D \eta \propto e^{u^{\dagger 2} P(u)}
$$

which gives $Z[J]$ as:

$$
Z[J]=\int e^{\int\left(u^{\dagger}\left(\partial_{t} u-\chi\left(-\partial_{L}\right) u\right)+u^{\dagger} u^{2}+u^{\dagger 2} P(u)+J u\right) d t d L} \operatorname{DuDiu}^{\dagger}
$$

with actions corresponding to (2.4), (2.5), (2.5) equals to

$$
\begin{gathered}
S=\int\left(u^{\dagger}\left(\partial_{t} u-\chi\left(-\partial_{L}\right) u\right)+u^{\dagger} u^{2}\right) d t d L \\
S=\int\left(u^{\dagger}\left(\partial_{t} u-\chi\left(-\partial_{L}\right) u\right)+u^{\dagger} u^{2}+u^{\dagger 2} u\right) d t d L \\
S=\int\left(u^{\dagger}\left(\partial_{t} u-\chi\left(-\partial_{L}\right) u\right)+u^{\dagger} u^{2}+u^{\dagger 2} u-u^{\dagger 2} u^{2}\right) d t d L
\end{gathered}
$$

From view of these generation functionals for different forms of Balitsky-Kovchegov hierarchy it is clear why (2.5) is referred as hierarchy with pomeron merging. From field theory point of view it contain addition interaction term. If we use analogy with charged scalar field theory interaction term in (2.4) can be called pomeron splitting term and additional term in (2.5) can be called pomeron merging term. Changed term in (2.6) does not impose additional symmetry to theory.

All three field theory has simple symmetry structure (translational invariance in $L$ and $t$ ). This is much simpler that BFKL field theory which is invariant under conformal transformation. It is easy to check that free motion equation has form of BFKL equation for all three field theories.

\section{BFKL reduction}

Balitky-Kovchegov hierarchy of equations is tree level approximation of so called BFKL field theory. It is therefore interesting to check if (2.10) corresponds in some sense to BFKL field theory generating fuctional.

BFKL field theory [11] can be written in form of functional integral [12]:

$$
Z_{B F K L}\left[J, J^{\dagger}\right]=\int D \Phi D \Phi^{\dagger} e^{S}
$$

where $S=S_{0}+S_{I}+S_{E}$ with terms corresponding to propagation of free BFKL pomerons, merging and splitting of BFKL pomerons, interaction with the target and projectile. Free action is given by:

$$
S_{0}=\int d y d^{2} x_{1} d^{2} x_{2} \Phi^{\dagger}\left(x_{1}, x_{2}, y\right) \nabla_{1}^{2} \nabla_{2}^{2}\left[\partial_{y}+H\right] \Phi\left(x_{1}, x_{2}, y\right)
$$


Pomeron interaction term is local in rapidity and is given by:

$$
\begin{array}{r}
S_{I}=\frac{2 \pi \bar{\alpha}_{s}^{2}}{N_{c}} \int d y \frac{d^{2} x_{1} d^{2} x_{2} d^{2} x_{3}}{x_{12}^{2} x_{23}^{2} x_{31}^{2}}\left[\left(L_{12} \Phi^{\dagger}\left(x_{1}, x_{2}, y\right)\right) \Phi\left(x_{2}, x_{3}, y\right) \Phi\left(x_{3}, x_{1}, y\right)+\right. \\
\left.\left(L_{12} \Phi\left(x_{1}, x_{2}, y\right)\right) \Phi^{\dagger}\left(x_{2}, x_{3}, y\right) \Phi^{\dagger}\left(x_{3}, x_{1}, y\right)\right]
\end{array}
$$

Term containing interaction with the target and projectile function is given by

$$
S_{E}=-\int d y d^{2} x_{1} d^{2} x_{2} \Phi\left(x_{1}, x_{2}, y\right) J\left(x_{1}, x_{2}, y\right)+\Phi^{\dagger}\left(x_{1}, x_{2}, y\right) J^{\dagger}\left(x_{1}, x_{2}, y\right)
$$

where differential operator $L_{12}=x_{12}^{4} \nabla_{1}^{2} \nabla_{2}^{2} . J\left(x_{1}, x_{2}, y\right), J^{\dagger}\left(x_{1}, x_{2}, y\right)$ represent projectile and target currents. From point of view of evolution BK equation $-J^{\dagger}\left(x_{1}, x_{2}, y\right)$ is initial condition for $\Phi\left(x_{1}, x_{2}, y\right)$. From structure of (3.1) it is not evident that (2.10) can be reduced from (3.1).

Attempts to obtain simpler generation functional was tried earlier in [13]. Obtained form is almost identical to (2.10) with few particularities. First one is that reduction was done by introductions of additional auxiliary fields into action. It can be shown that this can be avoided if we restrict ourselves to following currents:

$$
\begin{gathered}
J\left(x_{1}, x_{2}, y\right)=x_{12}^{2} \int_{0}^{\infty} k d k J_{0}\left(x_{12} k\right) j(k, y) \\
J^{\dagger}\left(x_{1}, x_{2}, y\right)=\nabla_{1}^{2} \nabla_{2}^{2}\left(x_{12}^{2} \int_{0}^{\infty} k d k J_{0}\left(x_{12} k\right) j^{\dagger}(k, y)\right)
\end{gathered}
$$

For these currents it can be shown that if we ignore second term in (3.3), $Z_{B F K L}\left[J, J^{\dagger}\right]$ can be represented as:

$$
Z_{B F K L}\left[J, J^{\dagger}\right]=Z^{\prime}\left[j, j^{\dagger}\right] Z^{0}
$$

where $Z^{0}$ is independent of $j$ and $j^{\dagger}$

Second one is that obtained Pomeron merging term has quite different structure (in notation of [13]):

$$
S_{\phi}=\frac{2 \alpha_{s}^{2} N_{c}}{\pi} \int d y d^{2} k\left(\nabla_{k}^{2} k^{4} \nabla_{k}^{2} \phi(k, y)\right) \phi^{\dagger}(k, y) \phi^{\dagger}(k, y)
$$

Such structure arises from structure of Pomeron merging vertex in (3.3):

$$
\left.\frac{1}{x_{12}^{2} x_{23}^{2} x_{31}^{2}} L_{12} \Phi\left(x_{1}, x_{2}, y\right)\right) \Phi^{\dagger}\left(x_{2}, x_{3}, y\right) \Phi^{\dagger}\left(x_{3}, x_{1}, y\right)
$$

However unlike Pomeron splitting vertex in (3.3) which was theoretically derived, structure of Pomeron merging vertex was conjectured in [11] to provide symmetry between target and projectile. It can be shown what we can introduce different Pomeron merging vertex:

$$
\left.\frac{1}{x_{12}^{2} x_{23}^{2} x_{31}^{2}} \Phi\left(x_{1}, x_{2}, y\right)\right) P_{23} \Phi^{\dagger}\left(x_{2}, x_{3}, y\right) P_{31} \Phi^{\dagger}\left(x_{3}, x_{1}, y\right)
$$

where $P_{i j}=\frac{1}{x_{i j}} L_{i j}^{-1} x_{i j}^{2}$ This vertex with redefinition of $\Phi^{\dagger}$ also gives theory symmetric between target and projectile. With this merging vertex (3.1) can be reduced to (2.10) up to renormalization. 


\section{Physical quantities}

Constructing field theory from (2.1) we ignored question of initial condition for $u(L, t)$ at $t=0$. For first order evolution equation we could replace initial condition with addition of current term $-J^{\dagger}(L, t)=u_{T}(L) \delta(t)$ in right-side of equation (2.1). Therefore generation functional $Z[J]$ can be rewritten as:

$$
Z\left[J, J^{\dagger}\right]=\int e^{\int\left(u^{\dagger}\left(\partial_{t} u-\chi\left(-\partial_{L}\right) u\right)+u^{\dagger} u^{2}+u^{\dagger 2} P(u)+J u+J^{\dagger} u^{\dagger}\right) d t d L} \operatorname{DuDiu}^{\dagger}
$$

Let's however consider projectile-target exclusive scattering process for projectile in eikonal approximation and rapidity $Y=\frac{T}{\bar{\alpha}_{s}}$. For scattering amplitude we can write

$$
S=\left.\frac{1}{Z_{0}} \sum \frac{\delta Z}{\delta^{i} J}\right|_{J=0} \frac{u_{P}^{n}(L)}{n !}=\frac{Z\left[u_{P}(L) \delta(t-T),-u_{T}(L) \delta(t)\right]}{Z_{0}}
$$

Therefore it can be argued that if we want to obtain scattering amplitude for exclusive scattering we should use currents:

$$
\begin{array}{r}
j(L, t)=-u_{T}(L) \delta(t) \\
j^{\dagger}(L, t)=u_{P}(L) \delta(t-T)
\end{array}
$$

or border condition:

$$
\begin{array}{r}
u(L, t=0)=u_{T}(L) \\
u^{\dagger}(L, t)=u_{P}(L)
\end{array}
$$

For generational functional $Z\left[J, J^{\dagger}\right]$ we can build classical equation of motion for fields $u, u^{\dagger}$.

$$
\begin{aligned}
\partial_{t} u & =\chi\left(-\partial_{L}\right) u-u^{2}-2 u^{\dagger} u \\
-\partial_{t} u^{\dagger} & =\chi\left(\partial_{L}\right) u^{\dagger}-u^{\dagger 2}-2 u^{\dagger} u
\end{aligned}
$$

In special case $u^{\dagger}=0$ which corresponds to limit case of dilute projectile it gives rise to original BK equation (1.1).

Using diffusive approximation with variable substitution(which represents traveling of wave)

$$
\chi_{0}=\gamma_{0} \chi_{0}^{\prime}, t=\frac{2 \tau}{\gamma_{0}^{2} \chi_{0}^{\prime \prime}}, L=\sqrt{\frac{\chi_{0}^{\prime \prime}}{\gamma_{0}^{2} \chi_{0}^{\prime \prime}}} x-2 \frac{\gamma_{0} \chi_{0}^{\prime \prime}-\chi_{0}^{\prime}}{\gamma_{0}^{2} \chi_{0}^{\prime \prime}} \tau
$$

we can get two non linear diffusion equations:

$$
\begin{array}{r}
\partial_{\tau} \phi=\partial_{x}^{2} \phi+\phi-\phi^{2}-2 \phi \phi^{\dagger} \\
-\partial_{\tau} \phi^{\dagger}=\partial_{x}^{2} \phi^{\dagger}+\phi^{\dagger}-\phi^{\dagger 2}-2 \phi^{\dagger} \phi
\end{array}
$$

Although simple solutions the form of traveling waves do exists (for example [14])

$$
\begin{array}{r}
\phi, \phi^{\dagger}=\frac{1}{2}\left(1 \pm \tanh (z)-\frac{1}{2 \cosh ^{2}(z)}\right) \\
z=\frac{x}{2 \sqrt{2}}-\frac{\tau}{4}
\end{array}
$$

they are unphysical (raise with momentum). 


\section{Conclusion}

In this work we obtained field theory for spatially homogeneous Balitsky-Kovchegov hierarchy there are many open questions left. Classical equations of motion can be constructed and have dilute projectile limit corresponding with original Balitsky-Kovchegov equation. Compared to Balitsky-Kovchegov equation full version of classical equations contain additional equation which corresponds to projectile evolution. This additional equation also introduces additional border condition. Although solutions for this equations is not known yet except for some unphysical cases, they could be solved at least numerically (work in progress) and used to construct scattering amplitude of spatially homogeneous target and projectile with Pomeron merging and splitting at least at tree level.

We thank A.V. Dmitriev, A.A. Popov and A.V. Abramovsky for useful discussions. The work is supported by RFBR grant 11-02-01395-a.

\section{References}

[1] M. Kozlov, E. Levin, and A. Prygarin, "The BFKL Pomeron Calculus in the dipole approach," Nucl. Phys. A792 (2007) 122-151, arXiv:0 704.2124 [hep-ph] .

[2] I. Balitsky, “Operator expansion for high-energy scattering,” Nucl. Phys. B463 (1996) 99-160, arXiv:hep-ph/9509348.

[3] I. Balitsky, "Factorization and high-energy effective action," Phys. Rev. D60 (1999) 014020, arXiv:hep-ph/9812311.

[4] Y. V. Kovchegov, "Small-x F2 structure function of a nucleus including multiple pomeron exchanges," Phys. Rev. D60 (1999) 034008, arXiv: hep-ph/9901281.

[5] Y. V. Kovchegov, "Unitarization of the BFKL pomeron on a nucleus," Phys. Rev. D61 (2000) 074018, arXiv:hep-ph/9905214.

[6] E. A. Kuraev, L. N. Lipatov, and V. S. Fadin, "The Pomeranchuk Singularity in Nonabelian Gauge Theories," Sov. Phys. JETP 45 (1977) 199-204.

[7] I. I. Balitsky and L. N. Lipatov, “The Pomeranchuk Singularity in Quantum Chromodynamics,” Sov. J. Nucl. Phys. 28 (1978) 822-829.

[8] A. H. Mueller, "Soft gluons in the infinite momentum wave function and the BFKL pomeron," Nucl. Phys. B415 (1994) 373-385.

[9] Unknown, “Unknown,".

[10] P. C. Martin, E. D. Siggia, and H. A. Rose, "Statistical dynamics of classical systems," Phys. Rev. A8 (1973) 423-437.

[11] M. Braun, "Conformal invariant equations for nucleus-nucleus scattering in perturbative QCD with N(c) to infinity," arXiv:hep-ph/0504002 [hep-ph].

[12] C. Contreras, E. Levin, and J. S. Miller, "BFKL Pomeron calculus: nucleus-nucleus scattering," Nucl.Phys. A880 (2012) 29-47, arXiv:1112.4531 [hep-ph].

[13] S. Bondarenko, "Langevin equation in effective theory of interacting QCD pomerons in the limit of large N(c)," Nucl.Phys. A792 (2007) 264-287, arXiv: hep-ph/0703045 [hep-ph] .

[14] D. Amati, G. Marchesini, M. Ciafaloni, and G. Parisi, "Expanding Disk as a Dynamical Vacuum Instability in Reggeon Field Theory," Nucl.Phys. B114 (1976) 483. 